\title{
Erratum zu: Mathematik der privaten Krankenversicherung
}

\section{Erratum zu:}

\section{T. Becker, Mathematik der privaten Krankenversicherung,}

Studienbücher Wirtschaftsmathematik, https://doi.org/10.1007/978-3-658-16666-3

Während der Produktionsphase des Titels ist versehentlich die Aktualisierung einer Literaturangabe auf Seite VI nicht vollständig vorgenommen worden.

Dies ist die korrekte Literaturangabe:

Milbrodt, H.: Aktuarielle Methoden der deutschen Privaten Krankenversicherung. Schriftenreihe Angewandte Versicherungsmathematik Heft 34. Verlag VVW, Karlsruhe, 2005.

Im Dezember 2016 erschien die erweiterte Neuauflage:

Milbrodt, H. und Röhrs, V.: Aktuarielle Methoden der deutschen Privaten Krankenversicherung. Aktualisierte Neufassung. Verlag Versicherungswirtschaft (VVW), 2. Auflage 2016.

Die aktualisierte Version des Buchfrontmatters kann hier abgerufen werden: https://doi.org/10.1007/978-3-658-16666-3 